\title{
Data Analysis of Ambient Intelligence in a Healthcare Simulation System: A Pilot Study in High-end Health Screening Process Improvement
}

\section{Ming-Shu Chen ( $\nabla$ tree1013@gmail.com )}

Oriental Institute of Technology https://orcid.org/0000-0002-2713-3546

Kun-Chih Wu

Chung Shan Institute of Science and Technology

Yu-Ling Tsai

National Taiwan University of Science and Technology

Bernard C. Jiang

National Taiwan University of Science and Technology

Research article

Keywords: health screening, healthcare simulation system, process improvement, service quality

Posted Date: October 5th, 2020

DOI: https://doi.org/10.21203/rs.3.rs-80964/v1

License: (c) (i) This work is licensed under a Creative Commons Attribution 4.0 International License.

Read Full License 


\section{Abstract}

This study aimed to reduce the total waiting time for high-end health screening processes. The subjects of this study were recruited from a health screening center in a tertiary hospital in northern Taiwan from September 2016 to February 2017 and a total of 2,342 high-end customers were collected. Arena software was used to simulate the examination process. We presented the simulation results of three different policies and compared those results to the current state. The first policy presented a predetermined proportion of customer types, in which the total waiting time was increased from 72.29 to 83.04 mins. The second policy was based on increased bottleneck resources and provided significant improvement, with the total waiting time was decreased from 72.29 to 28.39 mins. However, this policy also caused the cost to increase dramatically, while lowering the utilization of this exam station. The third policy was adjusting customer arrival times, which reduced the waiting time significantly, as the total waiting time was reduced from 72.29 to 55.02 mins. Although the wait time of this policy was slightly longer than that of the second policy, the additional cost was much lower than the second plan.

Scheduled arrival intervals could help to reduce customer waiting time in the health screening department base on FIFO rule. The simulation model of this study could be utilized and the parameters could be modified to comply with different health examination centers to improve process and service quality.

\section{Introduction}

With the rapid developments of technology, the economy, and medical technologies, as well as increases in average incomes, average life expectancies have increased, thus, middle-aged individuals are paying more attention to preventive healthcare and health examinations. Recent statistics on average life expectancies have shown that the average life expectancy of Taiwanese has reached 80.69 years old (National Development Center, Taiwan Population Estimate, 2022), officially entering the WHO defined aged nation, it has increased year by year for the last 10 years, and approximately three years has been added, as compared with that in 2006 (Department of Statistics, Ministry of the Interior, 2019). From the evidence, by 2026, the proportion of the population aged 65 and over in Taiwan will reach $21 \%$, making Taiwan a super-aged society (Strong, 2019). In addition, the high mortality rate caused by malignant tumors has led to the growth of health consciousness. Malignant tumors are the top cause of death in Taiwan; patients dying of malignant tumors accounted for $28.2 \%$ of the total number of deaths in 2018 , which is an increase of $1.6 \%$ compared with that in 2017 (Ministry of Health and Welfare, 2019). Nevertheless, the early identification of malignant tumors with suitable treatment greatly increases the probability of survival. This has also given rise to more attention being paid to preventive health examinations.

With the improvement of economic conditions, the general public has gradually attached importance to healthcare service quality. In recent years, various large-scale hospitals and professional health screening centers have gradually purchased expensive medical instruments and devices to provide high-end health screenings. Higher-end health screenings are more expensive self-pay health checks that have recently been prevalent in Japan, South Korea, and Taiwan, including endoscopy and imaging check-up. In 
addition, they have introduced smart health examination environments, which not only meet the needs of the market but also satisfy the general public's expectations towards high-end health screenings. With the high maturity and wide application of ambient intelligence (Aml), its application in healthcare has also been increasing to meet critical patient needs. The idea of Aml in healthcare is to create a digital environment that provides intelligent technologies with sensitive, adaptive, and responsive tools to empower physicians and patients (Bravo et al. 2016). Aml includes infrastructure such as sensors and devices interconnected through a network, as well as software development for recognition, reasoning, and analyzing the collected data (Cook et al. 2009).

According to Bloomberg's ranking of the efficiency of health care, Taiwan is in eighth place among 56 economies (Miller and Lu 2018). Not only outpatient clinics and inpatient departments but also high-end health screening services faces fierce competition. Due to this competition, many customers of high-end health screenings have started to pay attention to health examination waiting times. Waiting times have a negative association with customer satisfaction in most service systems. In the case of the healthcare system, although they may not be the major factor in customer satisfaction, long waiting times lead to low satisfaction (Anderson et al. 2007). As more and more of the general public are inclined to choose medical service systems with high quality and short waiting times, the requirements for hospital management should not be limited to the reduction of costs (Moscelli et al. 2016). In order to improve the mixed registration of an orthopedic outpatient clinic, Lu et al. (2014) used a simulation method to reduce outpatient clinic waiting times and improved the waiting times of patients of on-site outpatient department (OPD) registration by $29 \%-36 \%$ and the waiting time for appointment scheduling by $61 \%-63 \%$, which also significantly improved the patients' satisfaction with the service. Weng et al. (2011) used simulation data to evaluate the potential bottlenecks in the emergency room (ER), improve patient flow, and reduce ER waiting times. Moreover, a simulation model can also be used to evaluate the different scenarios of nursing personnel and modify the model to create the most appropriate operating model. Wong et al. (2011) randomly simulated patient flow and surgical data to improve the service quality and efficiency of the ER. How to improve medical quality and reduce patient waiting times to further improve satisfaction has been an important and critical indicator in the decision-making of hospital management.

Most past studies used discrete event simulation (DES) technologies to solve problems such as the reduced satisfaction of patients caused by excessive wait times, the low utilization rate of expensive medical instruments and devices, or the lack of physicians caused by inefficient scheduling systems. As a randomized system, simulation has been comprehensively applied to various industries, and both the manufacturing and service industries use simulation as a research tool. In recent years, numerous studies have started to use simulation software to improve or optimize medical service processes, and the research scope has grown to include human resource allocation, outpatient or ward process planning, ER process planning, and bed or instrument facility planning, etc. (Günal and Pidd 2010; Zhang et al. 2018).

Among the great number of papers devoted to modeling DES in a healthcare system, simulations in emergency departments are the most popular topic. The emergency room (ER) is the busiest and most crowded area of a hospital, and improving the resources allocated to the ER might be the most pressing 
problem. Vanbrabant et al. (2019) reviewed the literature of ER operations from the field of operations research and provided a classification in terms of the key performance indicators (KPIs) and improvement strategies. Wang et al. (2009) performed a case study of an ER in a hospital in France and applied ARIS Toolset to analyze the patient visit process and arena software to model the patient flow. Zhao et al. (2015) applied the DES model to detect ER bottlenecks, used benchmarks to set an achievable target, and conducted process improvements according to the design of experiment (DOE) technique. Zeinali et al. (2015) reconfigured resource allocation through a simulation system to improve patient flow in the ER and reduce congestion. The critical requirements for the simulation model were the frequency of each route, the patient arrival rate, and service duration. Kuo et al. (2016) analyzed the patient flow of an ER and developed a DES model in which different policies such as increasing resources, reallocating resources, and using staggered shifts were considered. Aboueljinane et al. (2014) used a DES model to analyze emergency services and the performance of referring patients to an adequate care institution when necessary to improve the performance of emergency medical services and further improve the optional efficiency of emergency medicine. Venkatadri et al. (2011) used a DES model to make process improvements in the cardiac catheterization room and reduce the waiting times and turnaround times of outpatients and inpatients. Hahn-Goldberg et al. (2014) applied the DES model to analyze the [sufficiency] of the Neurovascular Unit at Toronto Western Hospital (TWH) in Toronto, Canada. In order to perform a further analysis of the department capacity, they applied Simul8 discrete event simulation software based on real patient flow data. Their result confirmed that 20 beds would be able to accommodate the demand. In addition, their results showed that there was still a $20 \%$ growth in the number of patients under the current capacity. Chen et al. (2015) proposed a policy optimization procedure for a simulation model in which five ultrasound rooms provided eight types of ultrasound services. To simplify the simulation model, they assumed that patients were seen by appointment only and arrived on time and that no walk-in patients are allowed, whereas only the service time was nondeterministic. They proposed four appointment scheduling policies that each had its own parameters, such as the scheduled arrival intervals and the number of patients per arrival. Then, an optimization process based on the scatter search and tabu search methods was applied to find the near-optimal parameters for each model. Shi et al. (2014) used two measurement indicators (How can a clinic effectively utilize its resources? How long does a patient need to wait?) and a multifactorial experimental design to investigate the effects of six parameters (arrival rate, non-display rate, delivery rate, new patient rate, number of repeated appointments, and nurse appointment rate) on system performance. Rahman et al. (2013) used FlexSim software to simulate the different types of patients at cancer outpatient clinics and reduce the total examination time spent by patients at a clinic. Brenner et al. (2010) used SIMUL8 software to analyze the data of service processes and flows in the ER to simulate and calculate the total service volume of ER patients.

\section{Materials And Methods}

\subsection{Materials}


This study collected all the data regarding the waiting time spent at each examination station by customers receiving high-end health screening services, in which the customers spent at least USD \$800 on receiving a health examination, in a tertiary hospital in northern Taiwan from September 2016 to February 2017. During this period, a total of 2,342 customers received high-end health screenings. After the deduction of weekends and national holidays, there were 112 working days; therefore, an average of 21 customers received high-end health screenings each day. The spaces used for examinations included examination rooms 1 24 (as shown in Figure 1 and Table 1).

Table 1 Names of various examination rooms on the health screening space floor plan

\begin{tabular}{|llll|}
\hline Room No. & Space Name & Room No. & Space Name \\
\hline 1 & Comprehensive Examination Room & 15 & Bone Density Examination Room \\
\hline 2,3 & Cardiac Ultrasound Room & 16 & X-ray Examination Room \\
\hline 5 & Otolaryngology Room & 17 & Information/Consultation Station \\
\hline 6 & Ophthalmology Examination Room & 18 & Vision Examination \\
\hline 7 & ECG Room & 19 & Questionnaire Completion Station \\
\hline 8 & Multifunction Examination Room & 20,21 & Gynecology Examination Room \\
\hline 9,10 & General Examination Room & 22 & Nursing Station \\
\hline 11 & Abdominal Ultrasound Room & 23 & Blood Pressure Measurement \\
\hline $12,13,14$ & Endoscopy Examination Room & 24 & Blood Drawing Station \\
\hline
\end{tabular}

The purpose of this study was to reduce waiting times and improve the utilization rate of medical facilities and physicians and/or nursing manpower. The customers were classified into six types, as summarized in Table 2, according to the health examination package they had selected. The package for the high-end health examination in Far Eastern Memorial Hospital (FEMH) was designated depending on the gender and age of the customers. The first three types were for female customers and the last three types were for male customers. In addition, customer type $X_{1}$ and $Y_{1}$ were used to represent customers over the age of 55 and customer type $X_{2}$ and $Y_{2}$ were used to represent customers under the age of 55 . The remaining types were regardless of age. According to the past experiences of health managers and the joint discussions of internal medical and nursing experts, the order of the optimized health examination process was as shown in last column of Table 2. The names and codes of various examination items are shown in Table 3 , in which examination $O$ and examination $S$ represents female customers and examination $T$ represents male customers. Compared to other customer types, $X_{3}$ and $Y_{3}$ received additional examination $U$.

Table 2 Examination processes of six customer types 


\begin{tabular}{|llll|}
\hline Type & Gender & Age & Health Examination Process \\
$X_{1}$ & female & $>55$ & $A \rightarrow B \rightarrow C \rightarrow D \rightarrow E \rightarrow F \rightarrow G \rightarrow H \rightarrow I \rightarrow J \rightarrow K \rightarrow L \rightarrow M \rightarrow N \rightarrow O \rightarrow P \rightarrow Q \rightarrow R \rightarrow S \rightarrow V$. \\
$X_{2}$ & female & $<55$ & $A \rightarrow B \rightarrow D \rightarrow E \rightarrow F \rightarrow G \rightarrow C \rightarrow H \rightarrow I \rightarrow L \rightarrow M \rightarrow J \rightarrow P \rightarrow K \rightarrow N \rightarrow O \rightarrow R \rightarrow Q \rightarrow S \rightarrow V$. \\
$X_{3}$ & female & all & $A \rightarrow H \rightarrow B \rightarrow D \rightarrow F \rightarrow E \rightarrow C \rightarrow H \rightarrow I \rightarrow J \rightarrow K \rightarrow U \rightarrow L \rightarrow M \rightarrow P \rightarrow N \rightarrow O \rightarrow S \rightarrow R \rightarrow Q \rightarrow V$. \\
$Y_{1}$ & male & $>55$ & $A \rightarrow B \rightarrow C \rightarrow D \rightarrow E \rightarrow F \rightarrow G \rightarrow H \rightarrow I \rightarrow K \rightarrow J \rightarrow L \rightarrow M \rightarrow N \rightarrow P \rightarrow Q \rightarrow R \rightarrow T \rightarrow V$. \\
$Y_{2}$ & male & $<55$ & $A \rightarrow B \rightarrow E \rightarrow F \rightarrow G \rightarrow D \rightarrow C \rightarrow K \rightarrow H \rightarrow I \rightarrow J \rightarrow L \rightarrow M \rightarrow N \rightarrow R \rightarrow P \rightarrow Q \rightarrow T \rightarrow V$. \\
$Y_{3}$ & male & all & $A \rightarrow G \rightarrow B \rightarrow C \rightarrow F \rightarrow D \rightarrow E \rightarrow H \rightarrow I \rightarrow J \rightarrow K \rightarrow U \rightarrow N \rightarrow L \rightarrow M \rightarrow P \rightarrow T \rightarrow R \rightarrow Q \rightarrow V$. \\
\hline
\end{tabular}

Table 3 Names and codes of various high-end health examination stations

\begin{tabular}{|c|c|c|c|}
\hline Code & Examination Item & Code & Examination Item \\
\hline A & Check-in at the front desk & $\mathrm{L}$ & $\begin{array}{l}\text { Gastrointestinal endoscopy with } \\
\text { anesthesia }\end{array}$ \\
\hline B & $\begin{array}{l}\text { Changing health examination clothes } \\
\text { (dressing) }\end{array}$ & M & Endoscopic anesthesia recovery \\
\hline C & $\begin{array}{l}\text { Confirmation of data, completion of a } \\
\text { questionnaire }\end{array}$ & $\mathrm{N}$ & Thyroid ultrasound \\
\hline D & $\begin{array}{l}\text { Basic measurement of height and } \\
\text { weight }\end{array}$ & 0 & Female breast ultrasound \\
\hline$E$ & Vision, hearing & $P$ & Lunch \\
\hline $\mathrm{F}$ & Measurement of blood pressure & Q & Consultation with ophthalmologist \\
\hline G & Blood drawing & $\mathrm{R}$ & Consultation with otolaryngologist \\
\hline $\mathrm{H}$ & ECG examination & S & $\begin{array}{l}\text { Consultation with gynecologist and } \\
\text { obstetrician }\end{array}$ \\
\hline I & Abdominal ultrasound & $\mathrm{T}$ & Consultation with urologist \\
\hline$J$ & Cardiac ultrasound & U & CT of the coronary artery \\
\hline K & $\begin{array}{l}\text { Low-dose computed tomography of } \\
\text { the lung screening }\end{array}$ & V & $\begin{array}{l}\text { Leaving the medical center after } \\
\text { completing the examinations }\end{array}$ \\
\hline
\end{tabular}

\subsection{Data collection}

This study presented a preliminary study on the application of Aml, in which the data to be analyzed were received from smart wristbands combining RFID and a Wi-Fi tag worn by customers receiving health screenings. When the customers checked-in, the front desk staff would first collect their basic information. After the customers connected their wristbands to the RFID sensor at the door of the clinic, 
the system could then automatically recognize their identity and enter the time. When the subjects entered the clinic and exited it after the examination, the sensor would sense their wristband. Therefore, the data on the time spent in the examination could be collected.

After filtering and screening the data, this study obtained the complete and actual data needed to calculate the time spent by customers on each examination item. The screening criteria included: (1) screening according to the six designated customer types and hypothetical conditions; (2) deletion of records when customers missed the sensor while either entering or exiting the clinic room; (3) deletion of extreme values due to examination times exceeding two times the mean.

For the six customer types screened in this study, there were a total of 2,342 valid samples. According to the statistics of the customers' arrival time and the distribution of the number of customers, only 16 customers checked in before 7:00 AM, while most of the customers $(1,222$ in total) checked in between 7:00 and 8:00 AM. The number of customers checked in before 8:00 AM accounted for $52.2 \%$, followed by a total of 877 customers checking in between 8:00 and 9:00 AM (37.4\%). The customer daily arrival times were concentrated between 7:30 and 8:30 in the morning.

\subsection{Methods}

This study used Arena simulation software (ARENA 14.5, 2016) to perform analyses and collect various data, such as the actual examination time spent at various examination stations and the human resources required. This study used system simulation analysis to collect the data of six common customer types and the health examination process to develop a model conforming to the current status of the health examination institution in this study. Moreover, this study implemented different improvement strategies and proposed various system simulation models to evaluate whether the waiting time could be reduced and whether the utilization rate of medical resources could be improved, in order to provide the research results as the basis for high-end health screening centers to evaluate their decisionmaking. Few studies have investigated the application of simulation systems to improve the scheduling and wait times of high-end health screenings. This study could serve as a pilot study to facilitate the subsequent development of relevant studies.

\subsection{Simulation assumptions}

In consideration of the examination process requirements and the limitations of the examination space and examination items of the health examination center (room No. as table 1), a number of assumptions were made for the health examination process simulation: (1) there were at least 30 customers scheduled for a health examination every day; (2) the patient or customers dispatch method at the waiting area was simulated according to the FIFO rule (first in first out); (3) the ophthalmology examination room (No. 5) was a shared clinic for the examination items, in which basic examination item $D$ was performed before 12 o'clock noon and examination item E was performed after 12 o'clock noon; (4) one of the abdominal ultrasound rooms (No. 9) was a shared clinic for the examination items, in which male customers only received a thyroid ultrasound examination (examination item $\mathrm{N}$ ), while female customers received both 
examination items I and N; and (5) one of the abdominal ultrasound rooms (No. 10) was a shared clinic for the examination items, in which examination item / w performed before 12 o'clock noon and male customers received examination item $T$ after 12 o'clock noon.

\subsection{Simulation process limitations}

There have been very few relevant studies on the simulation of health examination processes. The many simulation limitations, rather than a lack of actual data, could result in dependency and a cause-andeffect relationship among different examination items. The limitations of the simulated health examination process in this study were as follows: (1) the customers needed to fast before having their blood drawn or receiving abdominal ultrasound and endoscopy examinations; (2) blood drawing, ECG examinations, abdominal ultrasounds, cardiac ultrasounds, and lung cancer screening needed to be performed before endoscopic examinations; (3) abdominal ultrasounds needed to be performed before cardiac ultrasounds; (4) female customers needed to concurrently receive thyroid ultrasound and breast ultrasounds during ultrasound examinations, as well as receive gynecology ultrasounds and new pap smears at the gynecology and obstetrics clinic; (5) male customers needed to receive prostate ultrasounds at the urology clinic; and (6) the computed tomography examinations of the coronary artery needed to be scheduled the day before, as it requires an expensive medical device that cannot be idle.

Based on the module setting of the constructed model, this study developed a simulation model conforming to the study site, where the study design was based on a model to divide individuals into different types of customers entering the system at the same time point.

\subsection{Model development}

This study used Arena software to implement the conceptual model. Under the limitations of needing to conform to the current status of the subjects and meet the conditions of various examination items, this study used the collected actual time data to develop the simulation model for the health examination processes of the aforementioned six types of customers. Because the service hours for the research subjects started from check-in at 7:00 AM and ended at completion of the health examination at 4:00 PM, the working hours of the simulation was set at nine hours. The total number of replications was set at 50 times, and the time unit of the simulation was set as minutes.

\subsection{Model validation}

The verification and validation processes were conducted to ensure the model could accurately and relevantly represent the actual system. Frist, the system's behavior was confirmed by the director of the department as well as the doctors and nursing experts. Then, the average waiting time of the baseline model was compared to the real system. The results showed that the real parameters were within the $95 \%$ confidence interval of the output parameters from the model.

In order to develop simulation strategies, this study first analyzed the potential main causes of the prolonged wait times. In order to investigate the main causes of the prolonged wait times of customers 
and help set up improvement goals, after discussions with medical and nursing teams in the hospital, this study used a cause and effect diagram (Figure 2) to determine several important factors (marked as **) resulting in prolonged wait times, including the late arrival of customers receiving health examinations, insufficient medical and nursing manpower at bottleneck stations, and different types of health examinations, which could all be the main causes of prolonged wait times. Therefore, this study developed three main improvement strategies for simulation: (1) adjusting the mixture of customer types; (2) increasing human resources at bottleneck stations; and (3) adjusting customer arrival times.

\section{Results}

After the simulation, the data report was summarized in the following tables. Regarding the data of the average wait time and total examination time of the simulated customers (as shown in Table 4), Number Out represented the number of customers receiving a health examination who exited the process. An average of 21.22 customers received health examinations every day. Wait Time referred to the individuals' wait time, while Total Time was the total time spent by individuals in the process. The said three parameters were calculated from the customers who exited the process. The time unit was minutes. The simulation results showed that the average wait time of the X3 module was the shortest $(20.37$ mins). Overall, the average wait time and total examination time of the male customers were longer than those of the female customers. In addition, the wait time data of the examination items at various examination stations (as shown in Table 3), as analyzed by Arena, showed that Wait Time of various examination items, and the unit was minutes. Number Out was the number of customers waiting at the examination stations (as the table 4). As shown in Table 5, this study found that gastrointestinal endoscopy [L] was the major bottleneck examination station, followed by stations $[\mathrm{J}]$ and $[\mathrm{K}]$.

Table 4 Average waiting and total examination time of customers in the Arena system simulation

\begin{tabular}{|llll|}
\hline Customer Type & Number Out & Wait Time $(\mathrm{min})$ & Total Time (min) \\
\hline$X_{1}$ & 2.36 customers & 61.24 & 192.06 \\
\hline$X_{2}$ & 6.34 customers & 49.53 & 186.37 \\
\hline$X_{3}$ & 0.36 customers & 20.37 & 63.96 \\
\hline$Y_{1}$ & 4.4 customers & 66.60 & 194.41 \\
$Y_{2}$ & 7.64 customers & 66.67 & 197.99 \\
$Y_{3}$ & 0.86 customers & 48.89 & 139.20 \\
\hline
\end{tabular}

Table 5 Current status results at the waiting area of various examination items in the Arena system simulation 


\begin{tabular}{|lll|}
\hline Queue & Wait time (min) & Number Waiting \\
\hline Height, weight [D] & 0.1126 & 0.0055 \\
\hline Vision, hearing [E] & 0.0971 & 0.0047 \\
\hline Blood pressure measurement [F] & 0.0985 & 0.0048 \\
\hline Blood Drawing [G] & 0.1058 & 0.0051 \\
\hline ECG [H] & 0.0804 & 0.0038 \\
\hline Abdominal ultrasound [I] & 0.0507 & 0.0024 \\
\hline Cardiac ultrasound [J]* & $\star 15.4203$ & 0.6852 \\
\hline Low-dose CT scan of the lungs [K]* & $\star 8.7374$ & 0.3952 \\
\hline Coronary angiography [U] & 0.5673 & 0.0033 \\
\hline Gastrointestinal endoscopy [L]* & $\star 35.2242$ & $\star 1.5535$ \\
\hline Thyroid ultrasound [N] (Male) & 0.0338 & 0.0008 \\
\hline Thyroid ultrasound [N] (Female) & 0.0335 & 0.0007 \\
\hline Plus female breast ultrasound [O] & & \\
\hline Consultation at Division of Ophthalmology [Q] & 0.3249 & 0.0139 \\
\hline Consultation at Division of Otolaryngology [R] & 1.4155 & 0.0597 \\
\hline Consultation at Division of Gynecology and Obstetrics [S] & 0.5135 & 0.0096 \\
\hline Consultation of Division of Urology [T] & 0.5854 & 0.0146 \\
\hline
\end{tabular}

*denotes that the wait time exceeded five minutes and that the examination stations were regarded as bottleneck stations.

Based on the above Arena simulation results (Table 4-5) and the cause and effect diagram of causes resulting in prolonged wait time (Figure 2), this study simulated six common customer modules. An average of 21.22 customers received health examinations every day. However, in order to meet the expectations of the study site (the hospital) to provide services to at least 30 customers receiving a health examination every day, and under the premise that the human resources at various examination stations would remain unchanged, the simulation results showed that the average wait time was increased from 52.22 minutes for 21 customers to 72.29 minutes for 30 customers. Therefore, this study confirmed that it was appropriate to develop the following three improvement strategies for the Arena simulation, including: (1) adjusting the mixture of customer types; (2) increasing bottleneck (gastrointestinal endoscopy exam [L]) resources; and (3) adjusting customer arrival times. The actual simulation results of the three improvement strategies are shown below. 
As the results of the Arena simulation analysis data show, the objective of this study was to increase the examination capacity from 21 to 30 customers. The simulation results of the three different policies were compared to the current state. The first policy was based on a predetermined proportion of customer types. Compared to the current state, the average wait time was reduced for four customer types; however, the wait time increased for the other two types. According to the simulation results, strategy 1 showed poor results, as the total wait time increased from 72.29 to 83.04 mins; however, strategies 2 and 3 resulted in effectively lower wait times. The total wait time was reduced from 72.29 to 28.39 mins for strategy 2 and from 72.29 to 55.02 mins for strategy 3. As the results of Table 6 show, overall, strategy 3 was the better solution.

Table 6 Average wait time and total examination time of each improvement strategy

\begin{tabular}{|lll|}
\hline $\begin{array}{l}\text { Improvement Strategy } \\
\begin{array}{l}\text { Increase daily customer number (from 21 } \\
\text { to 30) }\end{array}\end{array}$ & $\begin{array}{l}\text { Average total wait time } \\
(\mathrm{min})\end{array}$ & $\begin{array}{l}\text { Average total exam time } \\
(\mathrm{min})\end{array}$ \\
\hline Baseline of original condition & $\mathbf{7 2 . 2 9}$ & $\mathbf{1 8 8 . 2 1}$ \\
\hline (1) Adjust the mixture of customer types & 83.04 & 211.83 \\
\hline (2) Increase bottleneck [L] resources & 28.39 & 151.21 \\
\hline (3) Adjust customer arrival times & 55.02 & 171.48 \\
\hline
\end{tabular}

According to the simulation results, while strategy 2 had the best result, when resources were added to the bottleneck, the utilization rate would decrease and the cost would increase. Therefore, we suggested applying strategy 3 to decrease the waiting time. Follow-up researches could utilize the simulation model of this study and modify the parameters to comply with different health examination centers to improve the process and service quality. This study found that strategy 3 (adjusting customer arrival times) could be the best strategy to decrease total wait times.

\section{Discussion}

The results of studies simulating high-end health screening processes have rarely been published in international journals; however, numerous past studies have used the Arena system to simulate cases receiving healthcare processes. England and Roberts (1978), Lu et al. (2014), and Motola et al. (2013) mainly focused on the outpatient operating processes of different divisions, while Weng et al. (2011) and Van et al. (2019) mainly focused on the ER. Venkatadri et al. (2011) mainly focused on cardiac catheterization rooms, while Wong et al. (2011) mainly focused on operating room processes, and so on. In addition to Arena software, other studies have used various analysis software packages to simulate healthcare service processes. Rahman et al. (2013) used FlexSim software to simulate the different types of patients at cancer outpatient clinics and reduce the total examination time spent by patients at the 
clinic. Brenner et al. (2010) used SIMUL8 software to analyze the data of service processes and flows at an ER to simulate and calculate the total service volume of ER patients.

However, there is a lack of studies taking health examination centers as the research subjects, especially in regards to high-end health screening processes. The reason might be that more examination items and numerous stations are involved in current high-end health screening processes; thus, the conditions and limitations are also more complicated. Also, there is a lack of relevant smart facilities in most health examination centers for the collection of relevant data, such as examination times and wait times. Such limitations make it difficult to perform relevant studies.

According to the simulation results of the actual data of high-end health screenings, this study proposed the specific suggestions for the subsequent optimization of health examination processes, study sites (health examination centers), and future researchers interested in engaging in the simulation of a medical service process system. This study found, if the distribution of customer arrival times can be effectively controlled and late arrivals can be avoided, wait times can be effectively reduced and the total examination time can be shortened. These results were different from another study in health screening center with the application of reducing waiting time by using the Design for Six Sigma method in Taiwan (Wang et al. 2014). Therefore, subsequent researchers are advised to use the research model to apply multiple improvement strategies, as well as set up parameters for the model. Multiple objectives, such as cost reductions, wait time reductions, and decreasing the utilization rate of bottleneck station resources, could be jointly simulated to analyze their feasibility. Subsequent researchers are advised to simulate the optimized scheduling system and use the data collected in a real-time manner to develop a database to produce advice for optimized scheduling in a real-time manner according to the dynamic changes of different customers, in a manner similar to that of the instant feedback from Google Map route suggestions.

This study has the following two limitations. First, it investigated high-end health screenings, including painless gastrointestinal endoscopy with anesthesia [L], as well as coronary artery computed tomography $[\mathrm{U}]$, in the health examination processes of six types of customers according to their gender and age. These six processes were chosen because they could account for $80-90 \%$ of the service volume of the institution's high-end health screenings. However, other health examination processes were not analyzed, which is a limitation of this research. Second, as more service contents and examination items are involved in high-end health screenings, it is impossible to apply the research results to other institutions (hospitals or health examination centers) due to variations of the number of doctors, quantity of examination instruments and devices, spaces, or the number of staff. However, the research methods and simulation parameters could be provided as a reference for other institutions.

Finally, based on the above studies, this study summarized several important research findings. First, a simulation system can be effectively applied to large-scale hospitals where diversified examination items, complicated conditions and limitations, different examination plans, and types of customers receiving health examination can be simulated. It is believed that this simulation modules and study design could 
be applied to more medical units in hospitals, such as operating rooms. Second, the simulation results showed that, although the addition of devices or medical and nursing manpower can effectively reduce customer wait times, more funds and management costs are required. If customer arrival times can be effectively controlled or a batch check-in system can be developed, the wait times of customers receiving health examinations could be significantly improved. Third, the simulation can be applied to the preplanning of newly established health examination centers, site expansions, the planning of original units, new high-end health screening items, or simulation planning before the addition of new health examination packages to avoid unideal manpower, facility, or resource allocations, as well as poor process planning or facility planning. Although most health examination centers have considered improving the arrival rate of on-time arrivals or developing batch flow control at check-in, there is a lack of empirical data for reference. This study used actual wait time data to empirically prove the effectiveness of this strategy and propose two specific plans. It is believed that the results of this study could provide substantial assistance to health examination centers. This study proved that the development of a good check-in strategy could improve the wait time and medical utilization rate of customers receiving health examinations at health examination departments.

\section{Conclusions}

This study used FIFO to perform a simulation according to the developed model based on six improvement plans and three major improvement strategies for the waiting areas of various examination stations. The results showed that the effectiveness of adding human resources to bottleneck stations was the best (Table 6), followed by adjusting customer arrival times. Arrival time was found to be a key factor, which was similar to the result of Van et al. (2019), who prioritized emergency department arrivals. Triaging results in lower patient waiting time for patients in the higher priority acuity class, but longer waiting time for patients in the lowest priority class, which do not require immediate care and are not subject to FIFO and first come first service (FCFS).

According to the simulation results, this study reached two main conclusions and five specific research contributions: First, the effect of adjusting the proportions of customer types on reducing wait times was limited. In addition, as the number of customers receiving health examinations every day was different, the proportions of customer types varied. Although the use of a reservation system could control the number of different types of customers making a reservation in advance, the effectiveness of the simulation was not as expected. The second, we were adding resources to bottleneck stations and adjusting customer arrival times could effectively reduce wait times and total examination times. Providing that the number of different types of customers making a reservation is known, properly adjusting customer arrival times could make for a smoother process. If the process is still congested and wait times cannot be improved, human resources could be adjusted according to the situations of the health examination centers in various hospitals to allow manpower allocation to better conform to the daily composition of customer types. As the addition of human resources to bottleneck stations results in increased costs, adjusting customer arrival times may be the best choice for health examination centers to improve wait times. 
This study simulated the actual data of high-end health screenings to determine the adequate distribution of parameters and data, developed an improvement strategy model, and then simulated and analyzed the comparable data. The five specific research contributions were as follows. First, this study was the first to apply the Arena simulation system to simulate the comparable results of different types of high-end health screening processes. Second, the study site was a high-end health screening center. If there is a need to expand service volume or adjust the examination processes of different types of health examination packages, the simulation model in this study could help to evaluate and verify whether it is adequate. Third, the simulation model developed in this study could help identify the bottleneck stations of the service processes at various health examination centers to further improve the resources at these stations. Fourth, if there is a need to add manpower or expensive resources, such as instruments and devices, when faced with the expansion or addition of examination items, the simulation model in this study could help evaluate the quantity required to be added. Last, when the number of customers receiving high-end health screenings every day increases, this system could help evaluate whether the process and arrival times require adjustment, as well as whether the resource allocations of various examination stations require adjustment.

Although this was a pilot study, this study used the rule of thumb to choose six higher-order health examination processes for simulation, and the parameters were input according to the distribution of the actual health examination center data from the study site. Therefore, the simulation results are only for the reference of other health examination institutions. However, the common goals pursued by the health examination centers of various major hospitals and healthcare institutions is to reduce wait times, increase the number of customers receiving health examinations, and improve customer satisfaction. Therefore, the simulation model of this study could be further modified to meet the needs of different numbers of customers in different health examination centers, including arrival times, health examination processes, examination times, and different waiting intervals. It could even be used to perform simulations of different improvement strategies to achieve the objective of improving the quality of overall high-end health screenings.

With the rapid development of automation technology and artificial intelligence-related facilities, the application of simulation systems by medical institutions and the health industry could create significant market potential in the future. A simulation system could provide various medical units with services, including simulations of the allocation of human resources, the placement of beds, and process planning for patients at various departments, etc. The best improvement plans can be obtained through system simulation. Hopefully, subsequent researchers can further develop advanced models and appropriate simulation software functions for more comprehensive applications in the medical and health industry.

\section{Declarations}

\section{Acknowledgments}


This work was supported by Ministry of Science and Technology grant number MOST-106-2221-E-161004 \& MOST-105-2218-E-161-001 and Far Eastern Memorial Hospital grant number NSC-RD-104-1-12509. The authors would like to express their thanks for the participation and assistance of Dr. ChengMing Chiu (Direct of HMC), Dr. Yen-Ling Chiu (Nephrologist), Dr. Chi-Yu Yang (Cardiologist) and the medical teams of the Department of Health Management Center, Far Eastern Memorial Hospital.

\section{Authors' contributions}

M.-S.C. (Ming-Shu Chen) and B.C.J. (Bernard C. Jiang) conceived and designed the experiments; M.-S.C. and Y.-L.T. (Yu-Ling Tsai) performed the experiments; K.-C.W. (Kun-Chih Wu), and Y.-L.T. analyzed the data; B.C.J. and M.-S.C. were supervision; M.-S.C. contributed investigation material; Y.-L.T., K.-C.W., and M.-S.C wrote this paper; M.-S.C and K.-C.W. revised the final manuscript.

\section{Funding}

This research was funded by a grant from the Ministry of Science and Technology in Taiwan (MOST-1062221-E-161-004 \& MOST-105-2218-E-161-001). The authors declare no conflict of interest. The founding sponsors had no role in the design of the study; in the collection, analyses, or interpretation of data; in the writing of the manuscript, and in the decision to publish the results.

\section{Conflicts of interest/Competing interests}

The authors declare that they have no conflict of interest concerning this study.

\section{Availability of data and material}

This text is appropriate if the data are owned by a third party and authors do not have permission to share the data. The datasets generated during and/or analyzed during the current study are not publicly available, but are available from the corresponding author on reasonable request.

\section{Code availability}

Not applicable, it custom code.

\section{Ethics approval}

Not required, this is secondary data analysis and not including medical record information and patient privacy.

\section{Consent to participate}

Not applicable.

\section{Consent for publication}


This manuscript contains no person's details, images, or videos.

\section{References}

Aboueljinane L, Sahin E, Jemai Z, Marty J (2014) A simulation study to improve the performance of an emergency medical service: application to the French Val-de-Marne department. Simul Model Pract Theory 47:46-59.

Anderson RT, Camacho FT, Balkrishnan R (2007) Willing to wait? The influence of patient wait time on satisfaction with primary care. BMC Health Serv Res 7:31.

Bravo J, Cook DJ, Riva G (2016) Ambient intelligence for health environments. J Biomed Inform 64:207210.

Brenner S, Zeng Z, Liu Y, Wang J, Li J, Howard PK (2010) Modeling and analysis of the emergency department at University of Kentucky Chandler Hospital using simulations. J Emerg Nurs 36:303-310.

Chen PS, Robielos RAC, Palaña PKVC, Valencia PLL, Chen GYH (2015) Scheduling patients' appointments: Allocation of healthcare service using simulation optimization. J Healthc Eng 6:259-280.

Cook DJ, Augusto JC, Jakkula VR (2009) Ambient intelligence: Technologies, applications, and opportunities. Pervasive Mob Comput 5:277-298.

Department of Statistics, Ministry of the Interior (2019) Statistical analysis results of average lifespan of people in Taiwan. https://www.moi.gov.tw/stat/chart.aspx.

England W, Roberts SD (1978) Applications of computer simulation in health care. In Proceedings of the 10th Conference on Winter Simulation-Volume 2. IEEE Computer Society Press.

Günal MM, Pidd, M (2010) Discrete event simulation for performance modelling in health care: a review of the literature. J Simulation 4:42-51.

Hahn-Goldberg S, Chow E, Appel E, Ko FT, Tan P, Gavin MB, Ng T, Abrams HB, Casaubon LK, Carter MW (2014) Discrete event simulation of patient admissions to a neurovascular unit. J Healthc Eng 5:347-360.

Kuo YH, Rado O, Lupia B, Leung JM, Graham CA (2016) Improving the efficiency of a hospital emergency department: A simulation study with indirectly imputed service-time distributions. Flex Serv Manuf $J$ 28:120-147.

Lu TP, Tsai PF, Chu YC (2014) An agent-based collaborative model for orthopedic outpatient scheduling, in: In Computer Supported Cooperative Work in Design (CSCWD). Proceedings of the 2014 IEEE 18th International Conference on Computer Supported Cooperative Work in Design (CSCWD). 
Ministry of Health and Welfare, Executive Yuan (2019) statistical analysis results of main causes of death in 2018 in Taiwan. 2019. https://dep.mohw.gov.tw/DOS/Ip-4472-113.html.

Miller LJ, Lu W (2018) These are the economies with the most (and least) efficient health care. Bloomberg. com. September, 19.

Moscelli G, Siciliani L, Gutacker N, Gravellea HL (2016) Location, quality and choice of hospital: Evidence from England 2002-2013. Reg Sci Urban Econ 60:112-124.

Motola I, Devine LA, Chung HS, Sullivan JE, Issenberg SB (2013) Simulation in healthcare education: a best evidence practical guide. AMEE Guid No. 82 Med Teach 35:e1511-e1530.

National Development Council, Taiwan Population Estimate (2020) , Aging Timeline in 2019. https://www.ndc.gov.tw/Content_List.aspx?n=695E69E28C6AC7F3.

Rahman MM, Huggins A, Claudio DA (2013) Simulation study of an outpatient cancer clinic, Paper presented at the IIE Annual Conference.

Shi J, Peng Y, Erdem E (2014) Simulation analysis on patient visit efficiency of a typical VA primary care clinic with complex characteristics. Simul Model Pract Theory 47:165-181.

Strong M (2019) Taiwan will be a super-aged society by 2026. Taiwan News. https://www.taiwannews.com.tw/en/news/3636704.

Vanbrabant L, Braekers K, Ramaekers K, Van Nieuwenhuyse I (2019) Simulation of emergency department operations: a comprehensive review of KPIs and operational improvements. Comput Ind Eng 131:356-381.

Van Bockstal E, Maenhout B (2019) A study on the impact of prioritising emergency department arrivals on the patient waiting time. Health Care Manag Sci 22:589-614.

Venkatadri V Raghavan VA, Kesavakumaran V, Lam SS, Srihari K (2011) Simulation based alternatives for overall process improvement at the cardiac catheterization lab. Simul Model Pract Th 19:1544-1557.

Wang T, Guinet A, Belaidi A, Besombes B (2009) Modelling and simulation of emergency services with ARIS and arena. Case study: the emergency department of Saint Joseph and Saint Luc Hospital. Prod Plan Control 20:484-495.

Wang HMS, Wang SP, Lee W (2014) A case study for reducing client waiting time in a health evaluation center using design for Six Sigma. Engineering Management Journal, 26(2), 62-73.

Weng SJ, Tsai BS, Wang LM, Chang CY, Gotcher D (2011) Using simulation and data envelopment analysis in optimal healthcare efficiency allocations. In Proceedings of the Winter Simulation Conference. Winter Simulation Conference, 2011. 
Wong SY, Tsui KL, Chin KS, Xu M (2011) A simulation study to achieve healthcare service quality improvement in accident \& emergency department (AED). In Quality and Reliability (ICQR). 2011 IEEE International Conference.

Zhang X (2018) Application of discrete event simulation in health care: a systematic review. BMC Health Serv Res 18:687.

Zhao Y, Peng Q, Strome T, Weldon E, Zhang M, Chochinov A (2015) Bottleneck detection for improvement of emergency department efficiency. Bus. Process Manag J 21:564-585.

Zeinali F, Mahootchi M, Sepehri MM (2015) Resource planning in the emergency departments: A simulation-based metamodeling approach. Simul Model Pract Th 53:123-138.

\section{Figures}

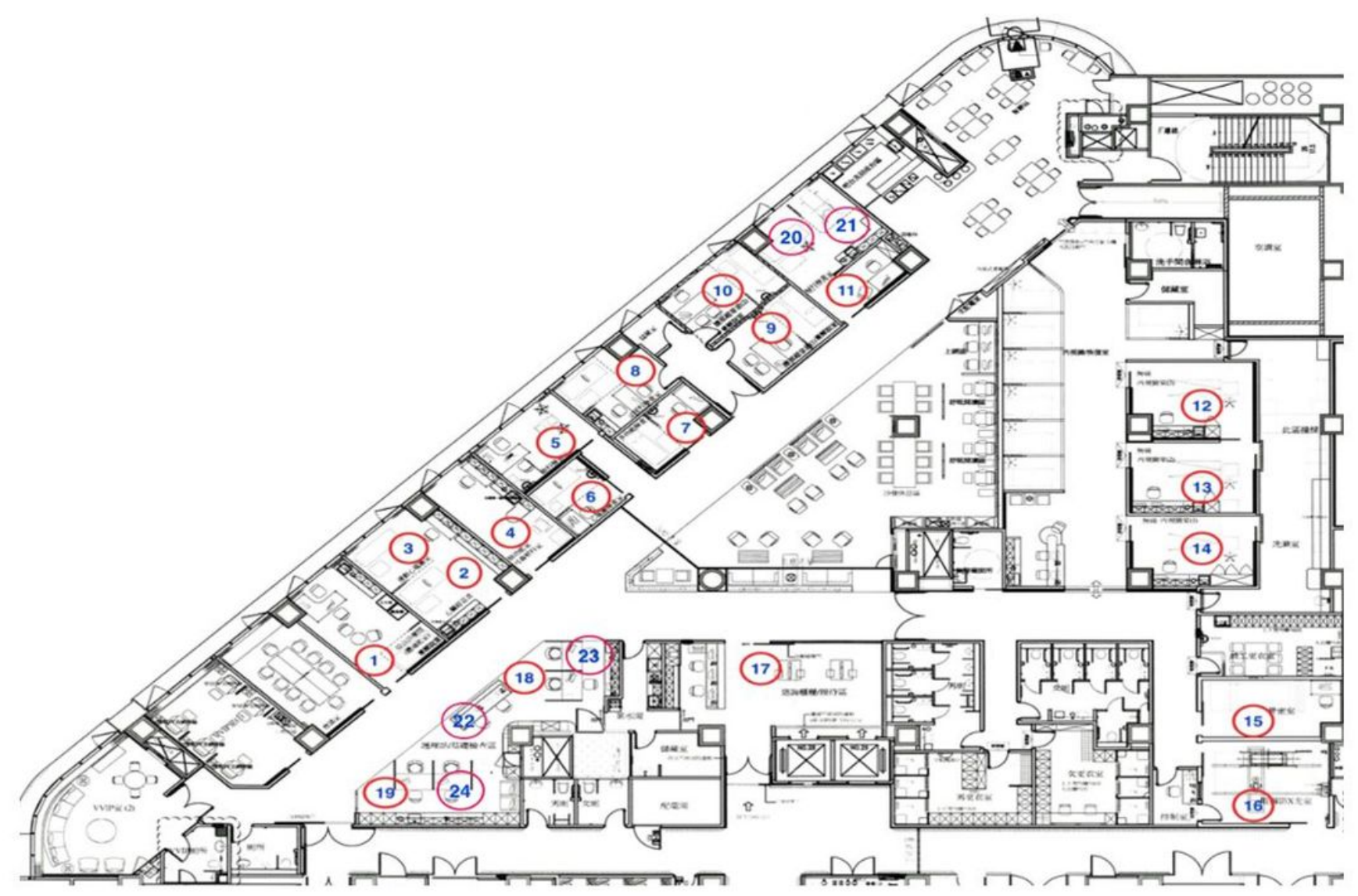

Figure 1

High-end health screening space floor plan and room number 


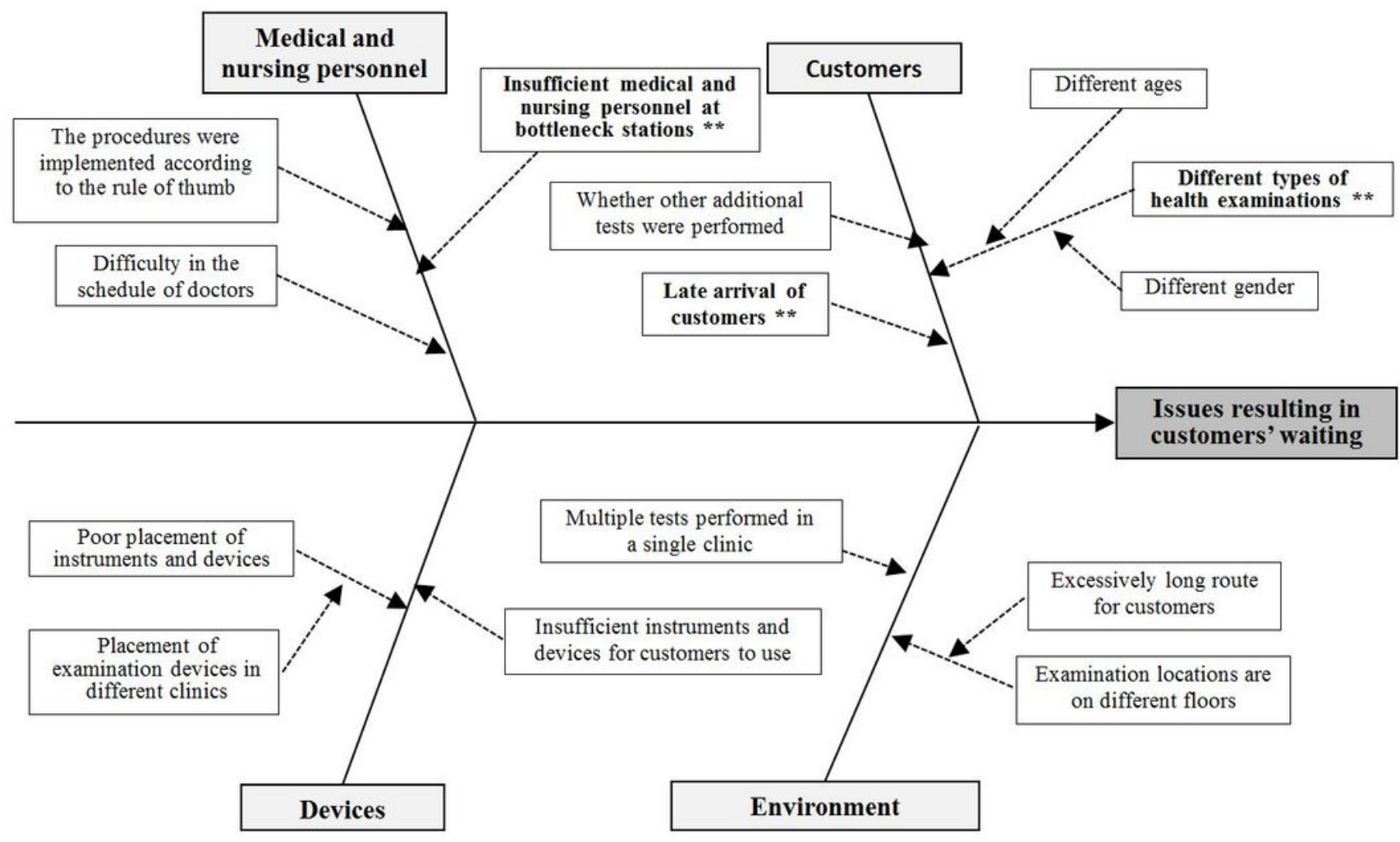

Figure 2

Cause and effect diagram of causes resulting in prolonged wait times 